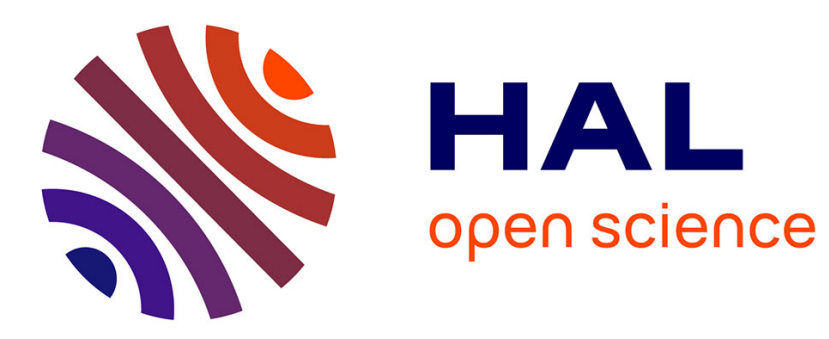

\title{
Action of counter-propagating laser beams on two-photon excitation of potassium vapour
}

T Efthimiopoulos, N Merlemis, M E Movsessian, D Pentaris, M Katharakis

\section{To cite this version:}

T Efthimiopoulos, N Merlemis, M E Movsessian, D Pentaris, M Katharakis. Action of counterpropagating laser beams on two-photon excitation of potassium vapour. Journal of Physics B: Atomic, Molecular and Optical Physics, 2010, 43 (5), pp.55401. 10.1088/0953-4075/43/5/055401. hal-00569871

\section{HAL Id: hal-00569871 https://hal.science/hal-00569871}

Submitted on 25 Feb 2011

HAL is a multi-disciplinary open access archive for the deposit and dissemination of scientific research documents, whether they are published or not. The documents may come from teaching and research institutions in France or abroad, or from public or private research centers.
L'archive ouverte pluridisciplinaire HAL, est destinée au dépôt et à la diffusion de documents scientifiques de niveau recherche, publiés ou non, émanant des établissements d'enseignement et de recherche français ou étrangers, des laboratoires publics ou privés. 


\title{
Action of counter-propagating laser beams on two- photon excitation of potassium vapour
}

\author{
T Efthimiopoulos ${ }^{1}, \mathrm{~N}$ Merlemis ${ }^{1,2}, \mathrm{M}$ E Movsessian ${ }^{3}$, D Pentaris ${ }^{1}$ and M \\ Katharakis ${ }^{1}$
}

1 Laser, Non linear and Quantum Optics Labs, Physics Department University of Patras, Patras, Greece 26500

2 Technological Educational Institute (TEI) of Athens, Department of Physics, Chemistry \& Materials Technology, Athens, Greece 12210

3 Institute for Physical Research, Armenian Academy of Sciences, Ashtarak-2, 37841, Yerevan Armenia

E-mail: nmerlemis@physics.upatras.gr, efthim@physics.upatras.gr

\begin{abstract}
We investigate the emissions of atomic potassium excited by two photons near the $4 \mathrm{~S}_{1 / 2}-6 \mathrm{~S}_{1 / 2}$ transition. Radiation near the $6 \mathrm{~S}_{1 / 2}-4 \mathrm{P}_{3 / 2,1 / 2}$ and $4 \mathrm{P}_{3 / 2,1 / 2}-4 \mathrm{~S}_{1 / 2}$ transitions are studied for the unidirectional and the bidirectional cases respectively. It is shown that the total output intensity increases considerably for the bidirectional propagation. This is attributed to the effective increase of the Doppler free excitation and the cancellation of the quantum interference effect that is associated with the multiple paths of excitation of certain states.
\end{abstract}

Keywords. Counter-propagating beams, amplified spontaneous emission (ASE), stimulated hyper Raman scattering (SHRS), parametric four-wave mixing (PFWM).

PACS: $32.80 . \mathrm{Wr}, 42.62 . \mathrm{Fi}, 42.65 . \mathrm{Re}, 52.35 . \mathrm{Mw}$

\section{Introduction}

The counter-propagation of laser beams in a non linear resonant medium can initiate some interesting parametric processes and produce axial or conical radiation [1]. In general the 
two-photon excitation near the S-S or S-D transitions of the alkali atoms causes various nonlinear processes, such as amplified spontaneous emission (ASE), stimulated hyper-Raman scattering (SHRS), parametric wave mixing (PWM) and two-step four-wave mixing (FWM) [2]. The competition among them has been examined in recent years [2-6]. Suppression phenomena can take place in such non-linear media because of the destructive quantum interference of the competing excitation pathways [7, 8]. In the following the processes involved are presented in some detail.

Figure 1 shows the energy level diagram of a potassium atom simplified to a fivelevel configuration. Two-photon excitation of the $6 \mathrm{~S}_{1 / 2}$ level can populate the $5 \mathrm{P}_{3 / 2,1 / 2}$ levels by either a SHRS or by an ASE process depending on the detuning of the two-photons from the $6 \mathrm{~S}_{1 / 2}$ level. In addition, two-photon excitation can lead to parametric emission of two photons at wavelengths near the $6 \mathrm{~S}_{1 / 2}-5 \mathrm{P}_{3 / 2,1 / 2}, 5 \mathrm{P}_{3 / 2,1 / 2}-4 \mathrm{~S}_{1 / 2}$ or the $6 \mathrm{~S}_{1 / 2}-4 \mathrm{P}_{3 / 2,1 / 2}, 4 \mathrm{P}_{3 / 2,1 / 2}-4 \mathrm{~S}_{1 / 2}$ transitions, due to phase matching conditions. Finally, the emissions corresponding to either ASE or SHRS processes can mix with the laser photons in a four-wave mixing (FWM) process to give a fourth photon. Efficient generation of this FWM radiation is done at different wavelengths near the $5 \mathrm{P}_{3 / 2,1 / 2}$ and $4 \mathrm{P}_{3 / 2,1 / 2}$ levels, compared to the emission of the parametric four wave mixing [4-6].

The spatial and temporal characteristics of the radiation produced from the above presented processes are different but difficult to discriminate. They can be though distinguished by their spectral characteristics. For example, by detuning the excitation from exact two-photon resonance $\left(\Delta_{4 S_{1 / 2}-6 S_{1 / 2}}\right)$ one can distinguish between ASE (on resonance) and SHRS (below resonance) emission. Additionally when the excitation frequency changes, the ASE frequency remains stable for a given transition, but the frequency of the SHRS changes. Also detuning far from resonance causes the ASE process to decrease drastically and the SHRS and the PWM processes dominate. It should be mentioned that the partially coherent ASE or the SHRS radiation could combine with the excitation laser radiation in a four-wave mixing process to produce a new radiation. This is a two-step process with the first step being the creation of the ASE or the SHRS photons and the second the mixing with the laser photons. It is understood that since the ASE process involves real population transfer and amplification there is a delay involved in the development of the radiation and consequently the produced pulse by FWM is delayed with respect to the parametrically emitted pulses. The delay depends primarily on the lifetime of the upper level which is involved in the ASE process and the amplification gain factor. 
An important phenomenon occurs when there is interaction between the processes involved. For example, ASE or SHRS radiations propagate in the forward (along the direction of the laser beam) and the backward direction but it was shown $[3,4]$ that the presence of the parametrically emitted radiation suppressed the forward components of ASE and SHRS while the backward ones were not affected. The parametric and the FWM processes are emitted only in the forward direction along the excitation laser direction.

In this paper we investigate the radiations (under the excitation of the $4 \mathrm{~S}_{1 / 2}-6 \mathrm{~S}_{1 / 2}$ twophoton transition of potassium atoms), which are emitted in the direction of the laser beam (forward- $\mathrm{I}_{\mathrm{FWD}}$ ) and in the opposite (backward- $\mathrm{I}_{\mathrm{BWD}}$ ) direction. We compare the results with the ones we observe when we have two counter-propagating laser beams in the medium. We show that using counter-propagating beams we are able to modify the atomic system response and increase the effective number of atoms that participate in the overall interaction.

\section{Experimental apparatus}

Figure 2, shows the experimental apparatus used in the experiment. An oscillator/amplifier dye laser (LUMONICS Hyperdye 300) was pumped by a $\mathrm{XeCl}$ excimer laser (LUMONICS HYPER-X), to excite the $4 \mathrm{~S}_{1 / 2}-6 \mathrm{~S}_{1 / 2}$ two-photon transition of potassium. The maximum dye laser energy available was $12 \mathrm{~mJ} /$ pulse and it was S-polarized. The laser radiation is equally split and directed inside the potassium vapour cell from opposite directions using a set of three partially reflecting (BS1) and highly reflecting mirrors (M'), which are carefully selected to equally divide the polarized dye laser beam into two counter-propagating beams of equal intensity (solid and dashed lines respectively). Optical path lengths (not shown equal in the figure for simplicity) and optical components inserted in the paths are carefully designed, ensuring that both laser beams entering the cell have the same properties (intensity, polarization, beam cross section etc.). Both beams are aligned properly for good beam overlapping in the cell. In the case where both counter-propagating laser beams are active (bidirectional case) the generated radiation exiting the cell was the same in each direction $\left(\mathrm{I}_{\text {Вотн }}\right)$. High reflecting mirrors $\mathrm{M}$ and one partially reflecting beam splitter BS1 are used to take the generated emission from each side of the cell into the monochromator. The optical paths of the emitted radiations from the two sides of the cell are carefully designed and calibrated (not shown in the graph for simplicity) in order to have comparable results. Experiments with one laser beam entering the cell (unidirectional case) were done using several beam stops were needed in order to allow only one of the two counter-propagating laser beams from entering the cell and also to allow only forward emitted radiation from the 
cell $\left(\mathrm{I}_{\mathrm{FWD}}\right.$, co-propagating with the laser beam) or only backward emitted radiation $\left(\mathrm{I}_{\mathrm{BWD}}\right.$, at opposite direction from the laser beam). The emitted radiation was resolved using a monochromator (Jobin Yvon H.R. 1000 M) and detected with a photomultiplier (HAMAMATSU R955). Interference filters (EDMUND SCIENTIFIC) centered at the emitted wavelengths and color-glass (MELLES GRIOT) filters were used to block the transmitted laser beam and to improve the signal to noise ratio. Averaging was done with a boxcar averager (SRS model SR250) and the signal was recorded with a computer using the SRS provided software. The spectral width of the laser pulse was $0.1 \mathrm{~cm}^{-1}$ while the pulse duration was $25 \mathrm{nsec}$. The potassium atoms were confined in a stainless steel heat pipe with a length of $17 \mathrm{~cm}$ and helium was used as a buffer gas. A temperature controller was used to vary the temperature in a range of $150{ }^{\circ} \mathrm{C}$ to $400{ }^{\circ} \mathrm{C}$ (which corresponds to an atomic density of $10^{13} \mathrm{~cm}^{-3}$ to $5 \times 10^{16} \mathrm{~cm}^{-3}$ respectively). The uniformity of the temperature within the $17 \mathrm{~cm}$ vapour zone was found to be better than $3 \%$, using three $\mathrm{J}$ type thermocouples. The laser frequency was tuned near the two-photon $4 \mathrm{~S}_{1 / 2}-6 \mathrm{~S}_{1 / 2}$ transition of potassium with a wave number $\mathrm{k}=13725.3 \mathrm{~cm}^{-1}$. Measurement of the ionization signal, due to resonant multiphoton ionization of the potassium atoms when the laser frequency was tuned at the two-photon $4 \mathrm{~S}_{1 / 2}-6 \mathrm{~S}_{1 / 2}$ transition, was also used in order to further verify the exact position of the twophoton transition.

\section{Results and discussion}

In this section we investigate the emission spectra of potassium atoms excited by two photons near the $4 \mathrm{~S}_{1 / 2}-6 \mathrm{~S}_{1 / 2}$ transition. We focus our analysis on the radiations generated when the $4 \mathrm{P}_{3 / 2}$ level is acting as the intermediate level, but similar results are observed for the other transitions. We set the two-photon detuning equal to $\Delta_{4 \mathrm{~S}_{1 / 2}-6 \mathrm{~S}_{1 / 2}}=2 \omega-\frac{E\left(6 \mathrm{~S}_{1 / 2}\right)-E\left(4 \mathrm{~S}_{1 / 2}\right)}{h}$

and we examine the features of the generated radiations adjusting $\Delta_{4 \mathrm{~S}_{1 / 2}-6 \mathrm{~S}_{1 / 2}}$, the cell temperature and the buffer gas pressure $\mathrm{P}_{\mathrm{bg}}$. First we discuss the forward (along the laser beam) and the backward emitted radiation case (opposite to the laser beam). Subsequently we present the results obtained with two counter-propagating laser beams in the cell.

It is well known that, due to the phase matching conditions, all the parametric and the wave mixing processes generate coherent radiation along the direction of the driving laser beam, while the coherent SHRS and the partially coherent ASE processes give radiation in 
the forward (along the direction of the laser beam) and also in the backward (opposite) direction of the laser beam. In addition, quantum interference phenomena occur when a certain excitation level can be reached by more than one route of excitation (for example the $6 \mathrm{~S}_{1 / 2}$ level excited by two laser photons and the same level excited by the $\omega_{\mathrm{IR}}$ and $\omega_{404}$ radiations produced simultaneously by the parametric process). The result is the reduction of the two-photon excitation. Also suppression of the SHRS and the ASE emissions in the forward (along the laser beam) direction is imposed by the presence of the parametric process as it was shown elsewhere [5-9], concerning the two-photon excitation to the $6 \mathrm{~S}_{1 / 2}$ level and the parametric generation through the $6 \mathrm{~S}_{1 / 2}-4 \mathrm{P}_{3 / 2}-4 \mathrm{~S}_{1 / 2}$ levels.

The reduction of the two-photon excitation and the suppression in the forward direction of the ASE or the SHRS are cancelled when the excitation of the atoms is done by two counter-propagating beams with opposite wave-vectors due to the non existence of the parametric process in this case. The Doppler free two-photon excitation of the $6 \mathrm{~S}_{1 / 2}$ state by the two counter-propagating beams causes the atoms to see always a frequency of $\omega_{L 1}+\omega_{L 2}=\omega+\omega-\left(k_{L 1}-k_{L 2}\right) V=2 \omega$ for $k_{L 1}=k_{L 2}$, independent of their velocity $V$ [10]. This is true when one photon comes from one beam and the second from the other. However when both absorbed photons belong to the same beam the above reasoning is not true. This Doppler-free case could be also seen as an indirect increase of the number density of the atoms without involving other complications (level broadening, phase mismatch, pump depletion) which occur when the atomic density is increased by raising the temperature of the cell. In order to examine the phenomenon we turn our attention to the observed emissions.

\subsection{Emission near the $693.9 \mathrm{~nm}\left(6 S_{1 / 2}-4 P_{3 / 2}\right.$ transition $)$}

The investigation was done for a narrow two-photon detuning region near the $4 \mathrm{~S}_{1 / 2}-6 \mathrm{~S}_{1 / 2}$ transition. The emission corresponding to the $6 \mathrm{~S}_{1 / 2}-4 \mathrm{P}_{3 / 2}$ transition in potassium at $693.9 \mathrm{~nm}$ propagates in both the forward and the backward directions for the unidirectional case. The intensity depends on the two-photon detuning, the excitation laser intensity and the atomic density of potassium vapour.

Figure 3 shows, for the unidirectional case the intensity $\mathrm{I}_{\mathrm{FWD}}$ of the $693.9 \mathrm{~nm}$ emission in the forward direction and the $\mathrm{I}_{\mathrm{BWD}}$ in the backward direction respectively as a function of the two-photon detuning $\Delta_{4 \mathrm{~S}_{1 / 2}-6 \mathrm{~S}_{1 / 2}}$ for a pulse intensity of $\mathrm{I}_{\mathrm{L}}=1.3 \mathrm{MW} / \mathrm{cm}^{2}$. The range of the two-photon detuning is within $0.15 \mathrm{~cm}^{-1}$ from resonance and the Doppler broadening is of the order of $0.01 \mathrm{~cm}^{-1}$. On the same graph we present the intensity of the $693.9 \mathrm{~nm}$ emission 
for the bidirectional case $\left(\mathrm{I}_{\text {ВОтH }}\right)$ with each beam having a pulse intensity of $\mathrm{I}_{\mathrm{L}}=1.3 \mathrm{MW} / \mathrm{cm}^{2}$. In the unidirectional case the forward $693.9 \mathrm{~nm}$ radiation intensity $\mathrm{I}_{\mathrm{FWD}}$ is larger than the $\mathrm{I}_{\mathrm{BWD}}$ and it is mainly parametric regardless of the two-photon detuning from resonance. The backward SHRS and ASE are not affected by the quantum cancellation effect. For the bidirectional case, we observe that the $693.9 \mathrm{~nm}$ radiation intensity $\mathrm{I}_{\mathrm{BOTH}}$ in each direction is higher than the total sum of the two intensities $\mathrm{I}_{\mathrm{SUM}}=\mathrm{I}_{\mathrm{FWD}}+\mathrm{I}_{\mathrm{BWD}}$ that should be expected if the two directions were acting independently, by more than a factor of two especially off resonance, for $\Delta_{4 \mathrm{~S}_{1 / 2}-6 \mathrm{~S}_{1 / 2}}$ between 0.10 and $0.15 \mathrm{~cm}^{-1}$.

These results are due to the quantum interference cancellation, since no wave mixing is possible in the case of counter-propagating beams, and the cancellation of the Doppler effect which brings more atoms in resonance with the field independently of their velocity. The effect is more pronounced, if the atomic density $\mathrm{N}$ is increased to $8 \times 10^{15} \mathrm{~cm}^{-3}$ as it is shown in figure $3(\mathrm{~b})$. We observe that the increase of the two-photon detuning range for the bidirectional case is considerable. Moreover, in both atomic densities, for the two-photon detuning of $0.15 \mathrm{~cm}^{-1}$, we recorded an increase of the intensity of the radiations by almost two orders of magnitude.

Additionally while the $\mathrm{I}_{\mathrm{FWD}}$ and the $\mathrm{I}_{\mathrm{BWD}}$ radiations in the unidirectional case have different origin (parametric in the forward direction and mainly SHRS in the backward) in the counter-propagation case both directions have radiations of the same origin (mainly SHRS).

In order to further investigate the origin of the radiation at $693.9 \mathrm{~nm}$ (ASE, SHRS or parametric) we checked the action of the buffer gas pressure $\mathrm{P}_{\mathrm{bg}}$ on the emission intensity in the unidirectional and bidirectional excitation cases, respectively. We expect that, due to the different characteristics of the ASE and SHRS processes, the action of the buffer gas should be different. The ASE is a two-step process and thus one could expect some increase of the ASE intensity when the buffer gas pressure is increased, since the increase of the collisions make the $6 \mathrm{~S}_{1 / 2}$ state broader, which helps to get more population to the upper level. On the other hand SHRS should decrease rapidly because collisions interrupt the one step coherent process. Finally the parametric emission intensity should only slightly change, because of the small impact of the buffer gas pressure $\mathrm{P}_{\mathrm{bg}}$ on the phase matching conditions, since the atoms of the buffer gas have no resonant transition close to the wavelengths studied.

In this experiment the parametric intensity is proved to be less dependent on the buffer gas pressure variation than the other processes. The results are presented in figure 4. It is evident that by increasing the buffer gas pressure up to $\mathrm{P}_{\mathrm{bg}}=50 \mathrm{mbar}$ the forward intensity 
of the $693.9 \mathrm{~nm}$ radiation for the unidirectional case practically does not change (parametric case). On the contrary, the intensity of the backward radiation (SHRS case) decreased by two orders of magnitude and the intensity for the bidirectional case seems to follow the SHRS emission change for high buffer gas pressure. Hence we could conclude that:

i) The backward radiation has a SHRS origin and therefore its intensity depends strongly on the buffer gas pressure $\mathrm{P}_{\mathrm{bg}}$. If it was of ASE origin we would even expect an increase of the intensity due to level broadening.

ii) The forward radiation is basically a parametric one left after the suppression of the forward SHRS by the parametric emission whose phase matching is affected to a smaller degree by the buffer gas pressure $\mathrm{P}_{\mathrm{bg}}$. This is probably due to the smooth change of the phase matching factor with buffer gas pressure.

iii) The nature of the emission in the bidirectional case seems to be SHRS, since the pressure dependence resembles the backward SHRS of the unidirectional case.

In Table 1 we provide a summary of indicative observations for the number density of $\mathrm{N}=9 \times 10^{15} \mathrm{~cm}^{-3}$ for two values of the laser intensity and for three values of the laser detuning from the two-photon resonance. It is obvious that for the case of zero two-photon detuning, the intensity of the $693.9 \mathrm{~nm}\left(6 \mathrm{~S}_{1 / 2}-4 \mathrm{P}_{3 / 2}\right)$ emission as seen in the forward direction increases by a factor of 5 when the input pulse intensity is doubled using two counter-propagating beams of $0.5 \mathrm{MW} / \mathrm{cm}^{2}$ intensity each one. The effect is stronger when the pumping laser intensity is $1 \mathrm{MW} / \mathrm{cm}^{2}$, where the emission at $693.9 \mathrm{~nm}$ increases by a factor of 8 in the bidirectional case, indicating the nonlinearity of the involved processes (as mentioned above in the case of the bidirectional excitation the dominant process is SHRS which is generated by a fifth-order nonlinear susceptibility). We should emphasize that for all laser intensities shown in table 1 , the emission intensity $\mathrm{I}_{\text {Вотн }}$ of the bidirectional set-up is much higher than the sum of the two intensities $\mathrm{I}_{\mathrm{FWD}}$ and $\mathrm{I}_{\mathrm{BWD}}$, that should be expected if the two directions were acting independently.

\subsection{Emission near the $766.5 \mathrm{~nm}\left(4 \mathrm{P}_{3 / 2}-4 S_{1 / 2}\right.$ transition $)$}

The emission near $766.5 \mathrm{~nm}$ for the unidirectional propagation of the pumping laser, at low laser intensity, was reported by Katharakis et al. [12]. The spectrum of the radiation has two main peaks: a high energy peak $\left(\mathrm{I}_{\mathrm{HEP}}\right)$ near $766.5 \mathrm{~nm}$ and a low energy peak $\left(\mathrm{I}_{\mathrm{LEP}}\right)$ near 766.6 $\mathrm{nm}$ respectively. According to a proposed model [12] the two peaks of the radiation near the $4 \mathrm{P}_{3 / 2}-4 \mathrm{~S}_{1 / 2}$ transition have a different origin. In particular the $\mathrm{I}_{\mathrm{HEP}}$ is a product of a parametric process along with the $693.9 \mathrm{~nm}$ emission. The second peak $\left(\mathrm{I}_{\mathrm{LEP}}\right)$ is the result of a two-step 
process which initially involves the creation of a radiation at $404 \mathrm{~nm}$ (transition at $5 \mathrm{P}_{3 / 2}-4 \mathrm{~S}_{1 / 2}$ ). This coherent radiation in sequence creates by a parametric process the $I_{L E P}$ and the other intermediate radiations corresponding to the transitions $5 \mathrm{P}_{3 / 2}-5 \mathrm{~S}_{1 / 2}-4 \mathrm{P}_{3 / 2}$ or $5 \mathrm{P}_{3 / 2}-3 \mathrm{D}_{3 / 2}-4 \mathrm{P}_{3 / 2}$. In the case of low intensity excitation of the $4 \mathrm{~S}_{1 / 2}-6 \mathrm{~S}_{1 / 2}$ two-photon transition the emission is observed only in the direction of the laser beam which is consisted with the proposed model.

Figure 5 shows, for the unidirectional excitation, a typical emission peak intensity (forward parametric $\mathrm{I}_{\mathrm{HEP}}$ spectral peak) recorded as a function of the two-photon detuning for a potassium density of $5 \times 10^{15} \mathrm{~cm}^{-3}$ and a low excitation intensity. As it was shown [12] there is some differentiation in the relative intensities of the $\mathrm{I}_{\mathrm{HEP}}$ and $\mathrm{I}_{\mathrm{LEP}}$ components with the twophoton detuning indicating the different generation processes involved (one-step and two-step processes correspondingly).

As the laser intensity increases above a few $\mathrm{MW} / \mathrm{cm}^{2}$ another broad peak appears close to the $\mathrm{I}_{\mathrm{HEP}}$ component $(766.5 \mathrm{~nm})$, which strongly depends on the laser intensity. This spectrally broad peak propagates in both directions and it is attributed to the ASE from the relaxing population of the $4 \mathrm{P}_{3 / 2}$ state. This state can be populated from the $5 \mathrm{P}_{3 / 2,1 / 2}$ state which is populated by the backward SHRS process or the cascade process. Resent theoretical calculations by Merlemis et al [11] indicate that under similar conditions, amplification without inversion (AWI) of the emitted ASE at the $4 \mathrm{P}_{3 / 2}-4 \mathrm{~S}_{1 / 2}$ transition is possible due to the existence of the strong fields in the $6 \mathrm{~S}_{1 / 2}-4 \mathrm{P}_{3 / 2}$ and $5 \mathrm{P}_{3 / 2}-4 \mathrm{~S}_{1 / 2}$ transitions respectively, providing a cascade or a V-type system.

Figure 6 shows the comparison of the unidirectional and bidirectional cases for the emission at $693.9 \mathrm{~nm}\left(6 \mathrm{~S}_{1 / 2}-4 \mathrm{P}_{3 / 2}\right)$ as a function of the laser peak intensity. Emission observed with counter-propagating beams has intensity much higher than that of the unidirectional case. This is true for both emissions at $693.9 \mathrm{~nm}$ and $766.5 \mathrm{~nm}$, respectively. The second emission is smaller than the first due to the strong $4 \mathrm{~S}_{1 / 2}-4 \mathrm{P}_{3 / 2}$ absorption. It is already mentioned above that for the unidirectional excitation case the parametric $4 \mathrm{P}_{3 / 2}-4 \mathrm{~S}_{1 / 2}$ emission is replaced by a strong bidirectional ASE when the laser intensity exceeds a limit. In the bidirectional case the $4 \mathrm{P}_{3 / 2}-4 \mathrm{~S}_{1 / 2}$ emission is again an ASE one, possibly without population inversion [11], while the $6 \mathrm{~S}_{1 / 2}-4 \mathrm{P}_{3 / 2}$ is a SHRS emission. We also observe that above certain excitation intensity saturation takes place. Recent theoretical simulations of the potassium atom as a four level system indeed predicts saturation for the $\mathrm{I}_{\mathrm{FWD}}$ as the result of the redistribution of the population among the excited states [13].

\section{Conclusions}


In this paper we presented the characteristics of the emissions observed in the case of the unidirectional and the bidirectional propagation of the excitation laser beam. We studied the emissions that correspond to the $6 \mathrm{~S}_{1 / 2}-4 \mathrm{P}_{3 / 2}(693.9 \mathrm{~nm})$ and the $4 \mathrm{P}_{3 / 2}-4 \mathrm{~S}_{1 / 2}(766.5 \mathrm{~nm})$ transitions, respectively. The results can be summarized as follows:

1) In the counter-propagating beam case the two-photon excitation tuning range and the intensity of the $6 \mathrm{~S}_{1 / 2}-4 \mathrm{P}_{3 / 2}$ radiation are considerably increased due mainly to the effective atomic population increase because of the Doppler free excitation.

2) In the unidirectional case the forward emitted radiation at $693.9 \mathrm{~nm}$ is mostly parametric while the backward is SHRS, due to the suppression of the forward SHRS by the parametric one.

3) In the counter-propagating case the $6 \mathrm{~S}_{1 / 2}-4 \mathrm{P}_{3 / 2}$ emission is mainly SHRS and much stronger than the emission of the unidirectional case. This is the result mainly of the Doppler free excitation, but also of the cancellation of the quantum interference effect since there is no parametric emission generated at the $4 \mathrm{P}_{3 / 2}-4 \mathrm{~S}_{1 / 2}$ transition that could suppress the SHRS emission.

4) There is a saturation for both $6 \mathrm{~S}_{1 / 2}-4 \mathrm{P}_{3 / 2}$ and $4 \mathrm{P}_{3 / 2}-4 \mathrm{~S}_{1 / 2}$ emissions for the unidirectional and the bidirectional cases above a certain excitation threshold due probably to the population redistribution.

\section{References}

[1] Vaicaitis V, Smilgevicius V and Piskarskas A 1999 Opt. Quantum Electr. 31, 263

[2] Merlemis N, Katharakis M, Koudoumas E and Efthimiopoulos T 2003 J. Phys. B: At. Mol. Opt. Phys. 36, 1943

[3] Lu M-H and Liu Y-M 1993 Appl. Phys. B. 57, 167

[4] Malcuit M S, Gauthier D J and Boyd R W 1985 Phys. Rev. Lett. 55, 1086

[5] Garrett W R, Moore M A, Hart R C, Payne M G and Wunderlich R 1992 Phys. Rev. A. 45,6687

[6] Boyd R W, Malcuit M S, Gauthier D J and Rzazewski K 1987 Phys. Rev. A. 35, 1648

[7] Moore M A, Garrett W R and Payne M G 1988 Opt. Commun. 68, 310

[8] Wunderlich R K, Garrett W R, Hart R C, Moore M A and Payne M G 1990 Phys. Rev A. 41, 6345

[9] Hart R C, Garrett W R and Payne M G 1992 Phys. Rev. A, 46, 4213

[10] Pomerantz A E and Zare R N 2003 Chem. Phys. Lett. 370, 515 
[11] Merlemis N, Lyras A, Katharakis M and Efthimiopoulos T 2006 J. Phys. B: At. Mol. Opt. Phys. 39, 1913

[12] Katharakis M, Merlemis N, Serafetinidis A and Efthimiopoulos T 2002 J. Phys. B: At. Mol. Opt. Phys. 35, 4969

[13] Armyras A, Pentaris D, Merlemis N, Lyras A and Efthimiopoulos T 2010 proceedings of the ICO-photonics, to be published 


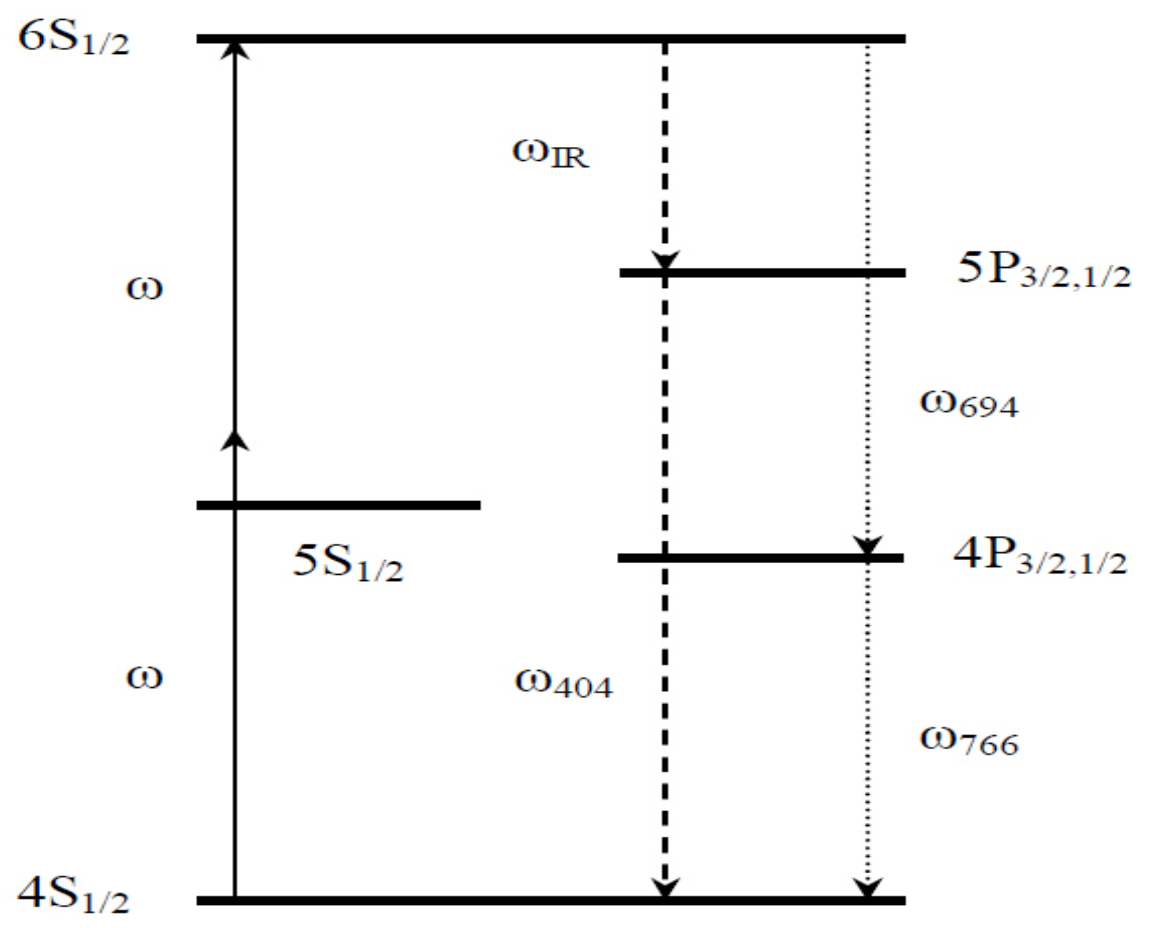

Figure 1. Energy level diagram of the potassium atom. In this five-level configuration the two-photon excitation field with frequency $\omega$ excites the atom to the state $6 \mathrm{~S}_{1 / 2}$. Parametric process produces $\omega_{\mathrm{IR}}$ and $\omega_{404}$ respectively. Absorption of two $\omega$ photons populates the $6 \mathrm{~S}_{1 / 2}$ state and subsequent or simultaneous one photon absorption can cause ionization. Radiation at $\omega_{\mathrm{IR}}$ could be also the result of an ASE or SHRS processes. 


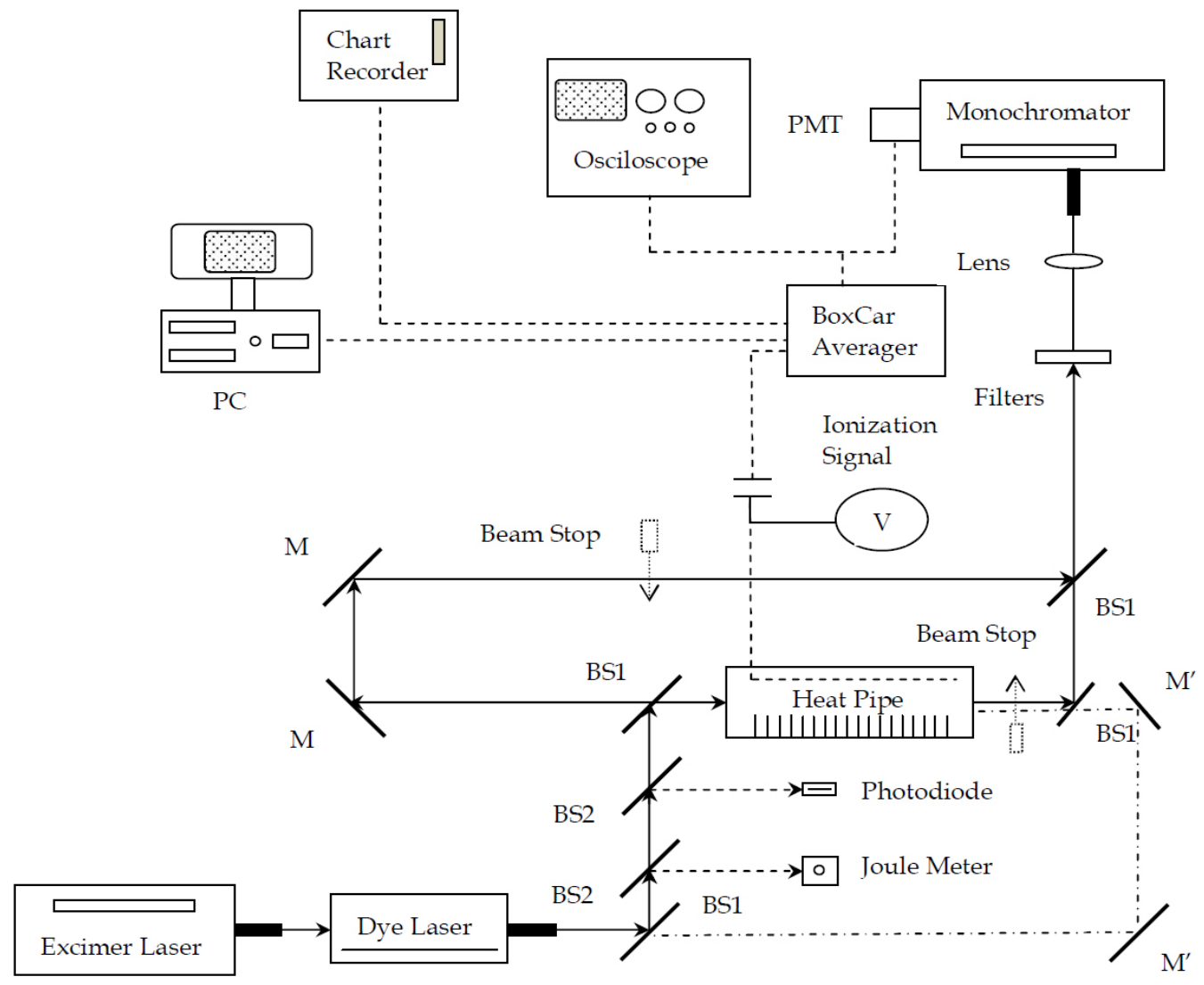

Figure 2. The simplified experimental set-up used in this work. Partially reflecting mirrors BS1 and high reflecting mirrors $\mathrm{M}$ and $\mathrm{M}^{\prime}$ are used to properly split the dye laser beam into two-counter-propagating beams and also to take emission measurements from both sides of the cell. Radiation leaking through BS2 partially reflecting mirrors is used for energy measurements as well as triggering of the systems. Interference filters and color-glass filters are used to block laser wavelengths from entering the monochromator for better signal to noise ratio and the ionization in the vapour cell is also recorded. 

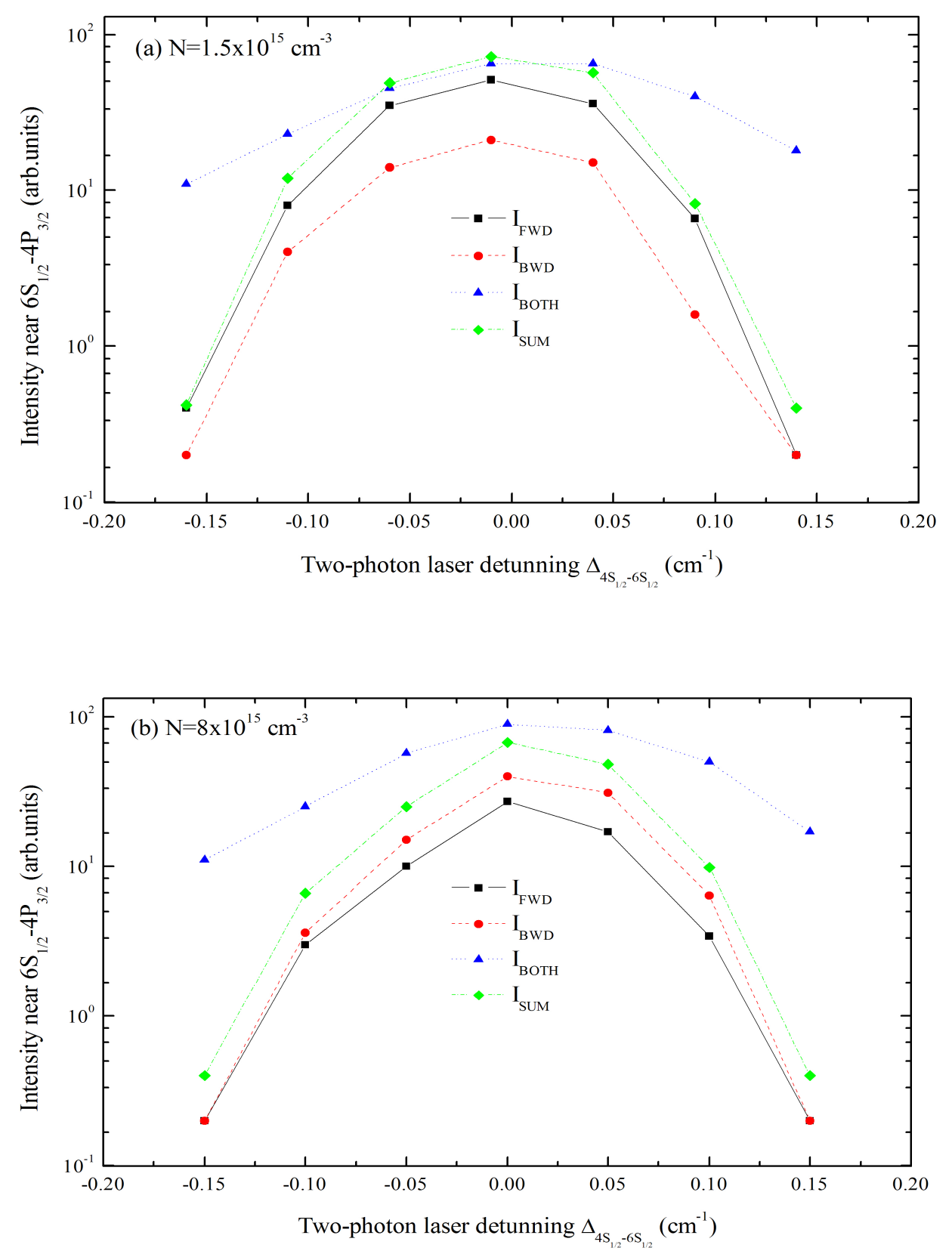

Figure 3. (a) Intensity of the emission near the $6 \mathrm{~S}_{1 / 2}-4 \mathrm{P}_{3 / 2}$ transition as a function of the twophoton detuning $\Delta_{4 \mathrm{~S}_{1 / 2}-6 \mathrm{~S}_{1 / 2}}$ for: the forward ( $\mathrm{I}_{\mathrm{FWD}}$ ) emitted radiation along the unidirectional beam, the backward $\left(\mathrm{I}_{\mathrm{BWD}}\right)$ emitted radiation, the sum $\left(\mathrm{I}_{\mathrm{SUM}}\right)$ of the previous two and the intensity of each component in the counter-propagation $\left(\mathrm{I}_{\mathrm{BO}}\right)$ case. Both the unidirectional and the counter-propagating cases were recorded with an excitation laser intensity of $1.3 \mathrm{MW} / \mathrm{cm}^{2}$ and a number density of $\mathrm{N}=1.5 \times 10^{15} \mathrm{~cm}^{-3}$. (b) The same graph as in (a) but for an elevated number density of $\mathrm{N}=8 \times 10^{15} \mathrm{~cm}^{-3}$. 


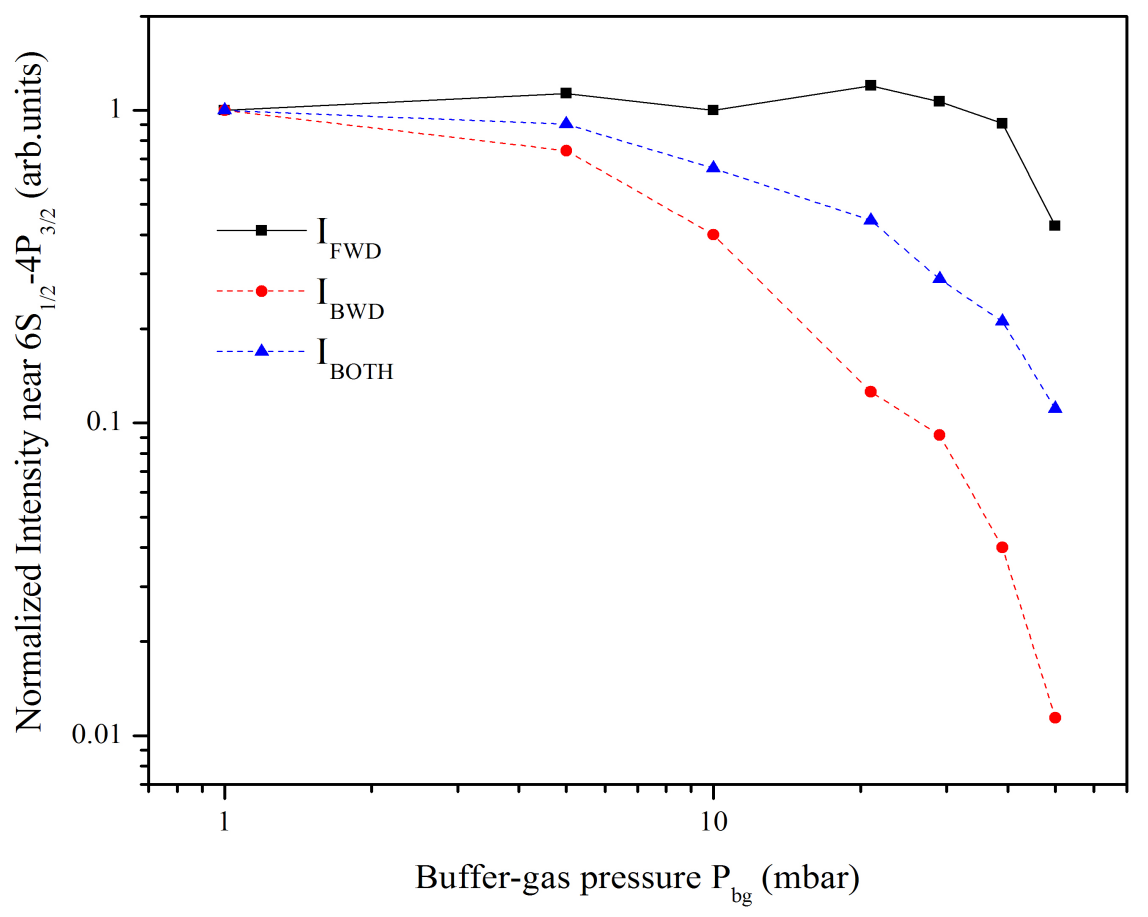

Figure 4. Normalized intensities of the $693.9 \mathrm{~nm}\left(6 \mathrm{~S}_{1 / 2}-4 \mathrm{P}_{3 / 2}\right)$ radiation as a function of the buffer gas pressure $\mathrm{P}_{\mathrm{bg}}$ for the unidirectional and the bidirectional (counter propagating) case of the two-photon excitation. The difference of the forward from the backward component is clear for pressures more than 10 mbar of helium. The backward $\left(\mathrm{I}_{\mathrm{BWD}}\right)$ component changes rapidly because it is produced by a SHRS process. The $\mathrm{I}_{\mathrm{FWD}}$ emission in the unidirextional case is mostly parametric. The component $\mathrm{I}_{\mathrm{BOTH}}$ corresponds to the counter-propagating case and its variation resembles that of the $\mathrm{I}_{\mathrm{BWD}}$ which is a SHRS emission strongly affected by the buffer gas pressure. 


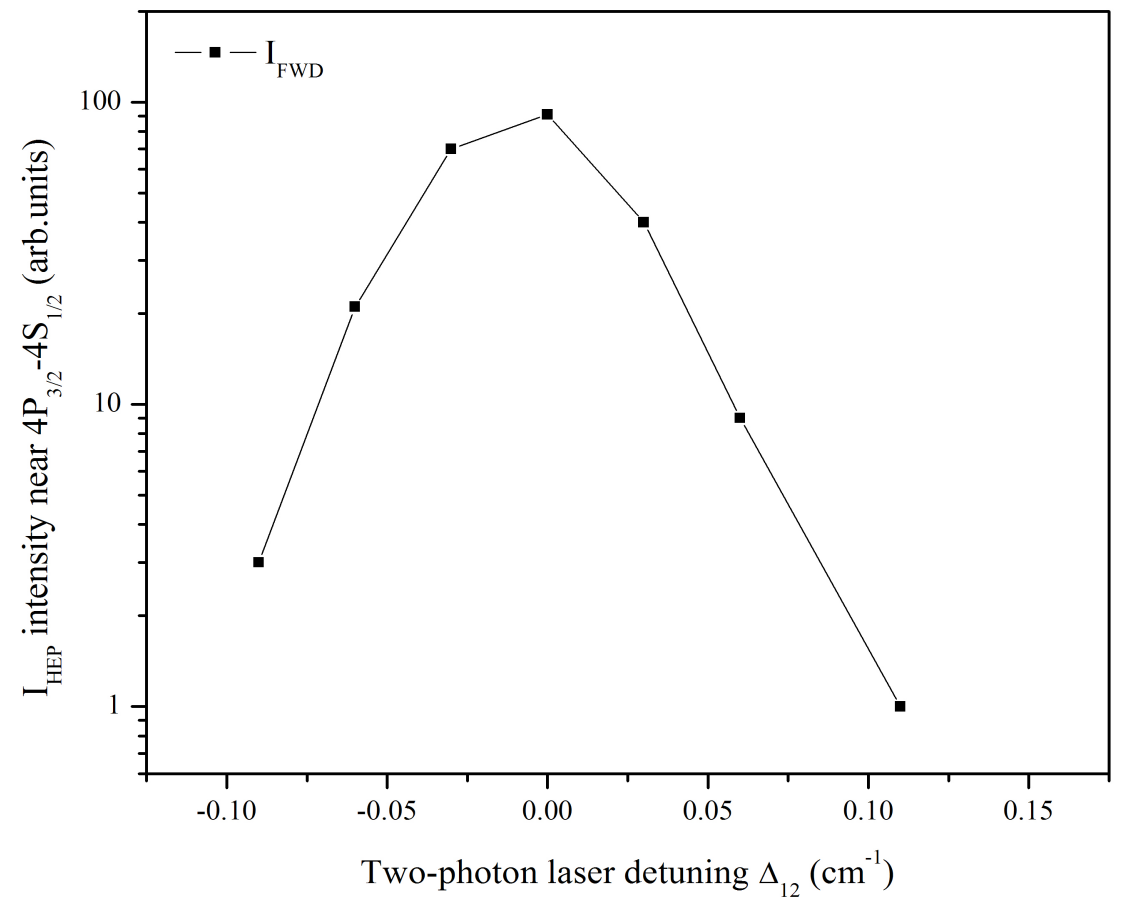

Figure 5. Intensity of the forward emitted parametric radiation $\left(I_{\mathrm{HEP}}\right)$ of the $4 \mathrm{P}_{3 / 2}-4 \mathrm{~S}_{1 / 2}$ transition (associated with the $693.9 \mathrm{~nm}$ emission of the $6 \mathrm{~S}_{1 / 2}-4 \mathrm{P}_{3 / 2}$ transition) as a function of the two-photon detuning $\Delta_{4 \mathrm{~S}_{1 / 2}-6 \mathrm{~S}_{1 / 2}}$, for the unidirectional case. The atomic density is $5 \times 10^{15} \mathrm{~cm}^{-3}$. 


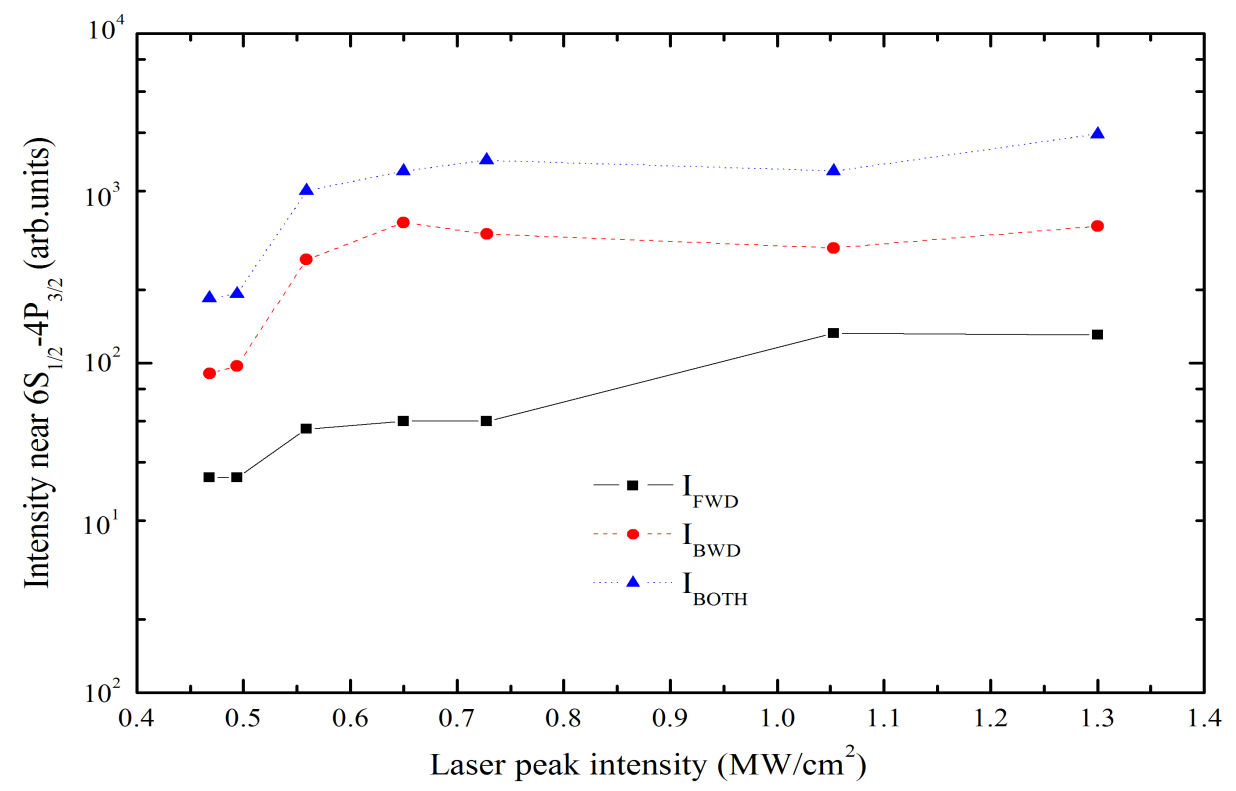

Figure 6. Intensity of the $693.9 \mathrm{~nm}\left(6 \mathrm{~S}_{1 / 2}-4 \mathrm{P}_{3 / 2}\right)$ as a function of the pumping laser intensity for the unidirectional case $\left(\mathrm{I}_{\mathrm{FWD}}\right.$ and $\mathrm{I}_{\mathrm{BWD}}$ ) and the bidirectional case $\left(\mathrm{I}_{\mathrm{BOTH}}\right)$ respectively. Above $0.6 \mathrm{MW} / \mathrm{cm}^{2}$ the emission appears saturated. 


\begin{tabular}{|c|c|c|c|}
\hline $\begin{array}{l}\text { Laser Direction } \\
\text { Observation point }\end{array}$ & $\begin{array}{c}\text { Intensity near } \\
6 \mathrm{~S}_{1 / 2}-4 \mathrm{P}_{3 / 2} \text { transition } \\
\text { (arb.units) }\end{array}$ & $\begin{array}{c}\text { Intensity near } \\
6 \mathrm{~S}_{1 / 2}-4 \mathrm{P}_{3 / 2} \text { transition } \\
\text { (arb.units) }\end{array}$ & $\begin{array}{c}\text { Intensity near } \\
6 \mathrm{~S}_{1 / 2}-4 \mathrm{P}_{3 / 2} \text { transition } \\
\text { (arb.units) }\end{array}$ \\
\hline & $\Delta_{12}=0.1 \mathrm{~cm}^{-1}$ & $\Delta_{12}=0 \mathrm{~cm}^{-1}$ & $\Delta_{12}=-0.1 \mathrm{~cm}^{-1}$ \\
\hline$\stackrel{0.5 \mathrm{MW} / \mathrm{cm}^{2}}{\longrightarrow} \underset{\text { FWD }}{\varnothing}$ & 2.3 & 4.0 & 3.2 \\
\hline 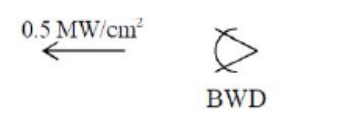 & 3.0 & 4.6 & 1.4 \\
\hline$\stackrel{0.5 \mathrm{MW} / \mathrm{cm}^{2}}{\longrightarrow 0.5 \mathrm{MW} / \mathrm{cm}^{2}} \underset{\mathrm{BOTH}}{\varnothing}$ & 13.0 & 20.0 & 12.0 \\
\hline$\varnothing_{\text {FWD }}^{8}$ & 3.6 & 5.6 & 7.4 \\
\hline 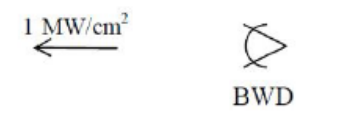 & 11.4 & 16.0 & 9.0 \\
\hline$\stackrel{1 \mathrm{MW} / \mathrm{cm}^{2}}{\longrightarrow} \stackrel{1 \mathrm{MW} / \mathrm{cm}^{2}}{\longleftrightarrow} \underset{\text { BOTH }}{\bigodot}$ & 40.0 & 45.0 & 38.0 \\
\hline
\end{tabular}

Table 1. Intensity of the $693.9 \mathrm{~nm}$ radiation at the $6 \mathrm{~S}_{1 / 2}-4 \mathrm{P}_{3 / 2}$ transition observed for two different laser excitation intensities with a number density of potassium equal to $\mathrm{N}=9 \times 10^{15}$ $\mathrm{cm}^{-3}$. The buffer gas pressure $\mathrm{P}_{\mathrm{bg}}$ was constant at 1.3 mbar. 